\title{
Three-zonal Wall Function for k- $\varepsilon$ Turbulence Models
}

\author{
M. Chmielewski ${ }^{1}$, M. Gieras \\ Warsaw University of Technology, Institute of Heat Engineering \\ Nowowiejska Street 21/25, 00-665 Warsaw \\ E-mail: chmielfam@wp.pl,marian.gieras@itc.pw.edu.pl
}

Received: 18 February 2013; revised: 19 March 2013; accepted: 2 April 2013; published online: 22 May 2013

\begin{abstract}
Most commercially available wall functions for $\mathrm{k}-\varepsilon$ turbulence models base on the two-zonal near-wall flow division assumption. Viscous and log-law sublayers are distinguished. In this article the three-zonal wall function concept with a buffer sublayer is developed. The aim of this new wall function is to improve the mean streamwise $U^{+}$velocity profile. The proposed wall function is validated on backward-facing step experimental data. Physical implications of the model performance are also discussed.
\end{abstract}

Key words: wall functions, turbulence modeling, near-wall treatment

\section{INTRODUCTION}

Turbulence flows are greatly impacted by wall boundaries [1], especially when dealing with flows through relatively narrow channels. Numerous experiments have shown that the near-wall flow can be divided into three sublayers: a viscous sublayer (a dimensionless distance from the wall $y^{+}<5$ ), where the mean velocity $U^{+}$shows a linear relationship with $\mathrm{y}^{+}[2]$, a fully-turbulent sublayer $\left(y^{+}>30\right)$ where the relationship between $U^{+}$(dimensionless velocity) and $y^{+}$ is approximated by logarithmic function [3], and a buffer sublayer between the two sublayers mentioned above. The aim of this article is to establish the analytical function describing $U^{+}$and $y^{+}$dependency in this sublayer, thus completing the system of equations for the whole near-wall $U^{+}$velocity. Some effort has been made to describe the Law of the Wall $\left(U^{+}\right.$and $y^{+}$dependency) by single formulas [4,5]. These attempts, however, have led to complicated functions that are inefficient in numerical calculations. The approach presented in this article assumes describing each of the sublayers separately, using simple analytic formulas. Such approach is planned to be used during initial turbulence calculations in a small turbine engine combustion chamber, whose complexity requires full $360^{\circ} 3$-D model. Such complex geometry requires a large grid, thus calculation using the proposed three-zonal wall function provides a very good initial guess of turbulence for further calculations.

CFD modeling of near-wall flows generally assumes no slip condition on walls, thus the gradients of solution variables, especially the tangential velocity, are large. To simulate near-wall turbulence directly (the "Low Re" approach) very fine meshes and modified turbulence models are required. Such fine meshes $\left(y^{+}\right.$around 1) together with 15-20 prism layers require large computational resources. To reduce the computational effort wall functions have been developed. In the wall function approach semi-empirical formulas are used to calculate flow parameters between the fully turbulent region and the wall. In this approach, in contrast to "Low Re", with refining the grid the results are deteriorating. Usually $y^{+}$below 15 is not recommended, because it causes large errors in wall shear stress and wall heat transfer calculations. In commercial CFD solvers, several wall functions depending on the turbulence model choice are implemented. This article considers the ANSYS FLUENT commercial code, but the proposed model can be implemented to any fluid dynamics numerical code with any turbulence model. In the FLUENT code four wall function models are available as default for the $\mathrm{k}-\varepsilon$ turbulence model [4]:

- Standard Wall Function

- Scalable Wall Function 
- Non-Equilibrium Wall Function

- Enhanced Wall Function

All of the above-listed wall functions are based on twozonal near-wall flow division, where the boundary layer is divided into viscous and log-law sublayer. Switch between those two zones occurs for specified $y^{+}$, in the FLUENT code this value was established as $y^{*}=11.225$. FLUENT uses wall unit $y^{*}$ instead of $y^{+}$and $U^{*}$ instead of $U^{+}$. In the equilibrium turbulent boundary layer these quantities are approximately equal. In general [8]:

$$
y^{*}=\frac{\rho C_{\mu}^{1 / 4} k_{p}^{1 / 2} y_{p}}{\mu}
$$

and

$$
y^{+}=\frac{\rho u_{\tau} y_{p}}{\mu}
$$

where:

$k_{p}$ - turbulence kinetic energy at the near-wall node $\mathrm{p}$,

$C_{\mu}$ - empirical constant $(=0.09)$,

$y_{p}$ - distance from point $\mathrm{P}$ to the wall,

$\mu$ - dynamic viscosity of the fluid,

$\rho$ - fluid molecular density,

$u_{\tau}-$ friction velocity.

The test case is the backward-facing step. Experimental data from Driver's and Seegmiller's research [6] has been used to validate the proposed three-zonal wall function implemented into the ANSYS FLUENT commercial CFD code.

\section{COMMERCIALLY AVAILABLE WALL FUNCTIONS}

\section{Standard Wall Function}

The standard wall function implemented in FLUENT, as well as in most commercial codes, is based on Lauder and Spalding [10] research. It is widely used in industrial flows modeling and given as a default option. As mentioned earlier, the boundary layer is divided into two sublayers. In the viscous sublayer $\left(y^{*}<11.225\right)$ laminar stress-strain relation is incorporated, thus:

$$
U^{*}=y^{*} \text {. }
$$

In the log-law sublayer $\left(y^{*}>11.225\right)$ logarithmic dependence between dimensionless velocity and wall unit is used:

$$
U^{*}=\frac{1}{\kappa} \ln \left(E y^{*}\right) .
$$

Dimensionless velocity:

$$
U^{*}=\frac{U_{p} C_{\mu}^{1 / 4} k_{p}^{1 / 2}}{\tau_{w} / \rho}
$$

where:

$U_{p}-$ mean velocity at node $\mathrm{p}$,

$\kappa \quad$ - von Karman constant $(=0.4187)$,

$E$ - empirical constant $(=9.793)$.

\section{Scalable Wall Functions}

The scalable wall function model prevents from deterioration of result when calculating on fine grids $\left(y^{*}<11\right)$. The main idea is to limit the $y^{*}$ to $y_{\text {limit }}^{*}=11.225$. This limitation demands the usage of log law, $y^{*}$ in (4) is replaced with:

$$
\tilde{y}^{*}=\operatorname{MAX}\left(y^{*}, y_{\text {limit }}^{*}\right)
$$

For grids coarser than $y^{*}>11.225$, obtained results are the same as for the standard wall function.

\section{Non-Equilibrium Wall Functions}

This type of wall function introduces sensitivity to pressure-gradient effects:

$$
\frac{\tilde{U} C_{\mu}^{1 / 4} k_{p}^{1 / 2}}{\tau_{w} / \rho}=\frac{1}{\kappa} \ln \left(E \frac{\rho C_{\mu}^{1 / 4} k_{p}^{1 / 2} y}{\mu}\right),
$$

where:

$$
\tilde{U}=U-\frac{1}{2} \frac{d p}{d x}\left[\frac{y_{v}}{\rho \kappa \sqrt{k}} \ln \left(\frac{y}{y_{v}}\right)+\frac{y-y_{v}}{\rho \kappa \sqrt{k}}+\frac{y_{v}^{2}}{\mu}\right]
$$

and:

$$
y_{v} \equiv \frac{\mu y_{v}^{*}}{\rho C_{\mu}^{1 / 4} k_{P}^{1 / 2}} ; y_{v}^{*}=11.225
$$

where:

$y_{v}-$ thickness of viscous sublayer.

\section{Enhanced Wall Treatment}

This wall function allows to resolve the viscous sublayer with very fine mesh (typically first near-wall node is around $y^{+} \approx 1$ ). Division into viscous and fully turbulent sublayers is based on the turbulent Reynolds number. This parameter is defined as:

$$
R e_{y} \equiv \frac{\rho y \sqrt{k}}{\mu},
$$

where $y$ is a distance between the wall and the cell center in normal direction:

$$
y \equiv \min _{\vec{r}_{w} \in \Gamma_{w}}\left\|\vec{r}-\vec{r}_{w}\right\|
$$

where $\vec{r}$ is the position vector at field point, $\vec{r}_{w}$ is position vector of the wall boundary and $\Gamma_{w}$ is the union of the walls. The limit value for this case is $R e_{y}^{*}=200$. The viscous sublayer is then completely resolved by the one-equation Wolfstein model. The fully turbulent layer is resolved based on the $\mathrm{k}-\varepsilon$ model.

Two-zonal near-wall flow division used in implemented wall functions results in imposed limitation on density of 
the computational grid. For standard and non-equilibrium wall functions the best practice is to locate each wall-adjacent cell's centroid within the viscous $\left(y^{+}<5\right)$ or the log-law sublayer $\left(30<y^{+}<300\right)$. Most desirable, however, is $y^{+} \approx 30$. With scalable and enhanced wall functions wall-adjacent cell should be placed on the order of $y^{+}=1$, but higher $y^{+}$is acceptable as long as it is well inside the viscous sublayer $\left(y^{+}<3\right.$ to 5$)$. In most cases of flow around complex geometries it is extremely hard (virtually impossible) to achieve uniform distribution of $y^{+}$around the whole geometry. This sometimes results in generation of areas with $5<y^{+}<30$, where all available wall functions are not valid.

\section{PROPOSED THREE-ZONAL WALL FUNCTION}

It has been proven in a number of experiments that the near-wall flow can be divided into three different regions called sublayers. In the most inner sublayer called a viscous sublayer (Fig. 1) the physical viscosity of the fluid plays the dominant role in momentum, heat and mass transfer. On the other hand, in the most outer sublayer, fully-turbulent, turbulence plays the dominant role. There is also a third region between the two previously mentioned, called a buffer sublayer, in which both effects: molecular viscosity and turbulence are crucial. Fig. 1, plotted in semi-log coordinates, illustrates this subdivision.

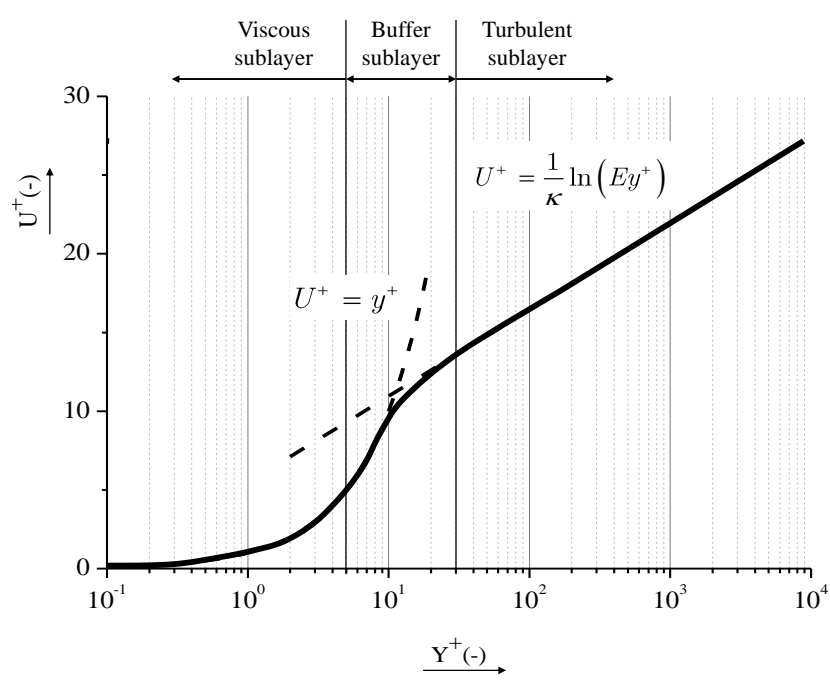

Fig. 1. Near-wall flow division

Function of the wall in the viscous sublayer:

$$
f_{1}\left(y^{+}\right)=U^{+}=y^{+} .
$$

In the log-law sublayer:

$$
f_{3}\left(y^{+}\right)=U^{+}=\frac{1}{\kappa} \ln \left(E y^{+}\right) .
$$

Those equations are the same as for the standard wall function, except for the region in which they cannot be used. As it can be seen in Fig. 1, the (12) can be used for $y^{+} \in(0 ; 5\rangle$ and (13) for $y^{+} \geq 30$ with a relatively small error. Outside these boundaries this function generates significant errors, which may affect the accuracy of numerical calculations of fluid flow parameters in devices with more complex geometries, such as compressor, turbine and turbine combustors with narrow channels.

The proposed approach is to describe function $f_{2}\left(y^{+}\right)$in the buffer sublayer with the use of the polynomial function. The order of such polynomial can be established by reviewing the available number of boundary conditions. It gives as a result the number of equations needed to determine individual polynomial coefficients $a_{n}$.

Note that there are two conditions for each function boundary. One of them describes a value of the function at boundary, and the second one describes slope of the function at boundary. They can be noted as a system of equations:

$$
\left\{\begin{array}{c}
\left.f_{2}\right|_{y_{1}^{+}}=\left.f_{1}\right|_{y_{1}^{+}} \\
\left.f_{2}\right|_{y_{2}^{+}}=\left.f_{3}\right|_{y_{2}^{+}} \\
\left.\frac{d f_{2}}{d y^{+}}\right|_{y_{1}^{+}}=\left.\frac{d f_{1}}{d y^{+}}\right|_{y_{1}^{+}} \\
\left.\frac{d f_{2}}{d y^{+}}\right|_{y_{2}^{+}}=\left.\frac{d f_{3}}{d y^{+}}\right|_{y_{2}^{+}}
\end{array}\right.
$$

Four boundary conditions indicate that function $f_{2}\left(y^{+}\right)$can be described by third-order polynomial:

$$
f_{2}\left(y^{+}\right)=a_{0}+a_{1} y^{+}+a_{2}\left(y^{+}\right)^{2}+a_{3}\left(y^{+}\right)^{3} .
$$

Calculating function derivative:

$$
\frac{d f_{2}}{d y^{+}}=a_{1}+2 a_{2} y^{+}+3 a_{3}\left(y^{+}\right)^{2} .
$$

Calculating derivatives from $f_{1}\left(y^{+}\right)$and $f_{2}\left(y^{+}\right)$:

$$
\begin{aligned}
\frac{d f_{1}}{d y^{+}} & =1 \\
\frac{d f_{3}}{d y^{+}} & =\frac{1}{\kappa y^{+}} .
\end{aligned}
$$

This system of equations can be noted in the matrix form:

$$
\left[\begin{array}{cccc}
1 & y_{1}^{+} & \left(y_{1}^{+}\right)^{2} & \left(y_{1}^{+}\right)^{3} \\
1 & y_{2}^{+} & \left(y_{2}^{+}\right)^{2} & \left(y_{2}^{+}\right)^{3} \\
0 & 1 & 2 y_{1}^{+} & 3\left(y_{1}^{+}\right)^{2} \\
0 & 1 & 2 y_{2}^{+} & 3\left(y_{2}^{+}\right)^{2}
\end{array}\right] \cdot\left[\begin{array}{c}
a_{0} \\
a_{1} \\
a_{2} \\
a_{3}
\end{array}\right]=\left[\begin{array}{c}
y_{1}^{+} \\
1 / \kappa \ln \left(E y_{2}^{+}\right) \\
1 \\
1 / \kappa y_{2}^{+}
\end{array}\right] .
$$


We can write:

$$
\left[\begin{array}{c}
a_{0} \\
a_{1} \\
a_{2} \\
a_{3}
\end{array}\right]=\left[\begin{array}{c}
y_{1}^{+} \\
1 / \kappa \ln \left(E y_{2}^{+}\right) \\
1 \\
1 /\left(\kappa y_{2}^{+}\right)
\end{array}\right] \cdot\left[\begin{array}{cccc}
1 & y_{1}^{+} & \left(y_{1}^{+}\right)^{2} & \left(y_{1}^{+}\right)^{3} \\
1 & y_{2}^{+} & \left(y_{2}^{+}\right)^{2} & \left(y_{2}^{+}\right)^{3} \\
0 & 1 & 2 y_{1}^{+} & 3\left(y_{1}^{+}\right)^{2} \\
0 & 1 & 2 y_{2}^{+} & 3\left(y_{2}^{+}\right)^{2}
\end{array}\right]^{-1} .
$$

Solution of the above system of equation is as follows:

$$
\begin{aligned}
& {\left[4\left(y_{2}^{+}\right)^{3} y_{1}^{+}-3\left(y_{2}^{+}\right)^{2}\left(y_{1}^{+}\right)^{2}-\left(y_{2}^{+}\right)^{4}\right] \cdot y_{1}^{+}+\left[-3\left(y_{1}^{+}\right)^{2}\left(y_{2}^{+}\right)^{2}+4\left(y_{1}^{+}\right)^{3}\left(y_{2}^{+}\right)-\left(y_{1}^{+}\right)^{4}\right] \cdot\left[\frac{1}{\kappa} \ln \left(E y_{2}^{+}\right)\right]} \\
& +\left(y_{1}^{+}\right)\left(y_{2}^{+}\right)^{4}-2\left(y_{1}^{+}\right)^{2}\left(y_{2}^{+}\right)^{3}+\left(y_{1}^{+}\right)^{3}\left(y_{2}^{+}\right)^{2}+\left[\left(y_{1}^{+}\right)^{2}\left(y_{2}^{+}\right)^{3}-2\left(y_{1}^{+}\right)^{3}\left(y_{2}^{+}\right)^{2}+\left(y_{1}^{+}\right)^{4}\left(y_{2}^{+}\right)\right] \cdot \frac{1}{\kappa y_{2}^{+}} \\
& a_{0}=\frac{4\left(y_{1}^{+}\right)\left(y_{2}^{+}\right)^{3}-6\left(y_{1}^{+}\right)^{2}\left(y_{2}^{+}\right)^{2}+4\left(y_{1}^{+}\right)^{3}\left(y_{2}^{+}\right)-\left(y_{1}^{+}\right)^{4}-\left(y_{2}^{+}\right)^{4}}{4} \\
& {\left[6\left(y_{1}^{+}\right)^{2}\left(y_{2}^{+}\right)-6\left(y_{1}^{+}\right)\left(y_{2}^{+}\right)^{2}\right] \cdot y_{1}^{+}+\left[6\left(y_{1}^{+}\right)\left(y_{2}^{+}\right)^{2}-6\left(y_{1}^{+}\right)^{2}\left(y_{2}^{+}\right)\right] \cdot\left[\frac{1}{\kappa} \ln \left(E y_{2}^{+}\right)\right]} \\
& a_{1}=\frac{+3\left(y_{1}^{+}\right)^{2}\left(y_{2}^{+}\right)^{2}-2\left(y_{1}^{+}\right)^{3}\left(y_{2}^{+}\right)-\left(y_{2}^{+}\right)^{4}+\left[3\left(y_{1}^{+}\right)^{2}\left(y_{2}^{+}\right)^{2}-2\left(y_{1}^{+}\right)\left(y_{2}^{+}\right)^{3}-\left(y_{1}^{+}\right)^{4}\right] \cdot \frac{1}{\kappa y_{2}^{+}}}{4\left(y_{1}^{+}\right)\left(y_{2}^{+}\right)^{3}-6\left(y_{1}^{+}\right)^{2}\left(y_{2}^{+}\right)^{2}+4\left(y_{1}^{+}\right)^{3}\left(y_{2}^{+}\right)-\left(y_{1}^{+}\right)^{4}-\left(y_{2}^{+}\right)^{4}} \\
& {\left[3\left(y_{2}^{+}\right)^{2}-3\left(y_{1}^{+}\right)^{2}\right] \cdot y_{1}^{+}+\left[3\left(y_{1}^{+}\right)^{2}-3\left(y_{2}^{+}\right)^{2}\right] \cdot\left[\frac{1}{\kappa} \ln \left(E y_{2}^{+}\right)\right]+2\left(y_{2}^{+}\right)^{3}} \\
& \frac{-3\left(y_{1}^{+}\right)\left(y_{2}^{+}\right)^{2}+\left(y_{1}^{+}\right)^{3}+\left[\left(y_{2}^{+}\right)^{3}-3\left(y_{1}^{+}\right)^{2}\left(y_{2}^{+}\right)+2\left(y_{1}^{+}\right)^{3}\right] \cdot \frac{1}{\kappa y_{2}^{+}}}{\left(y_{1}^{+}\right)\left(y_{2}^{+}\right)^{3}-6\left(y_{1}^{+}\right)^{2}\left(y_{2}^{+}\right)^{2}+4\left(y_{1}^{+}\right)^{3}\left(y_{2}^{+}\right)-\left(y_{1}^{+}\right)^{4}-\left(y_{2}^{+}\right)^{4}} \\
& {\left[2 y_{1}^{+}-2 y_{2}^{+}\right] \cdot y_{1}^{+}+\left[2 y_{2}^{+}-2 y_{1}^{+}\right] \cdot\left[\frac{1}{\kappa} \ln \left(E y_{2}^{+}\right)\right]+2\left(y_{1}^{+}\right)\left(y_{2}^{+}\right)} \\
& a_{3}=\frac{-\left(y_{1}^{+}\right)^{2}-\left(y_{2}^{+}\right)^{2}+\left[2\left(y_{1}^{+}\right)\left(y_{2}^{+}\right)-\left(y_{2}^{+}\right)^{2}-\left(y_{1}^{+}\right)^{2}\right] \cdot \frac{1}{\kappa y_{2}^{+}}}{4\left(y_{1}^{+}\right)\left(y_{2}^{+}\right)^{3}-6\left(y_{1}^{+}\right)^{2}\left(y_{2}^{+}\right)^{2}+4\left(y_{1}^{+}\right)^{3}\left(y_{2}^{+}\right)-\left(y_{1}^{+}\right)^{4}-\left(y_{2}^{+}\right)^{4}} .
\end{aligned}
$$

Since $y_{1}^{+}$and $y_{2}^{+}$are constant values, respectively equal to 5 and 30 as well as $\kappa$ and $E$, the three-zonal law of the wall can be written as:

$$
\left\{\begin{aligned}
y^{+}<5: & f_{1} & =y^{+} \\
y^{+} \in\langle 5 ; 30): & f_{2} & =-1.1297+1.4676 \cdot y^{+} \\
& & -0.0515 \cdot\left(y^{+}\right)^{2}+0.0006 \cdot\left(y^{+}\right)^{3} \\
y^{+} \geq 30: & f_{3} & =\frac{1}{\kappa} \ln \left(E y^{+}\right) .
\end{aligned}\right.
$$

Fig. 2 shows the course of dimensionless velocity near the wall for the proposed three-zonal model related to the course of two-zonal model used in the standard wall function. It can be observed that within the transition zone relative difference between both functions can be about $13 \%$.

The proposed three-zonal wall function has been successfully programmed in $\mathrm{C}$ language and implemented into the ANSYS FLUENT code.

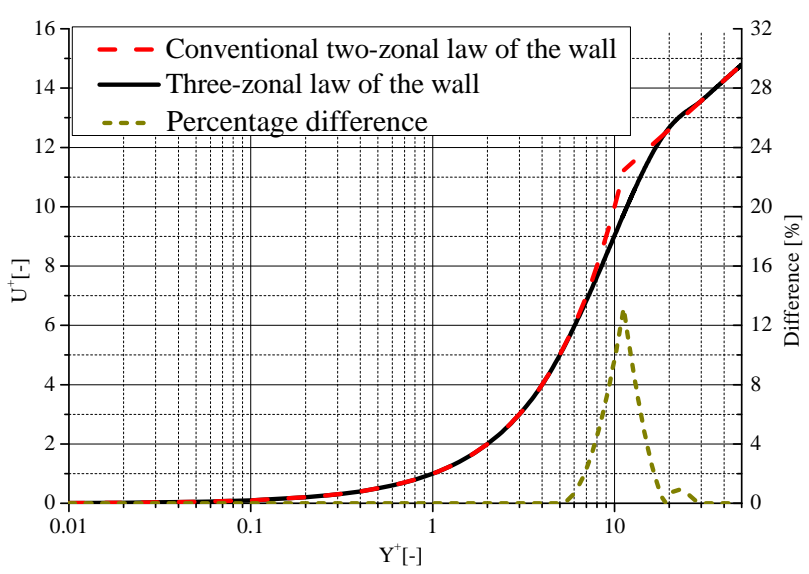

Fig. 2. Comparison between conventional and three-zonal wall function 


\section{RESULTS AND DISCUSSION}

Since scalable and enhanced wall functions require $y^{+}$ around 1 , the occurrence of areas of $5<y^{+}<30$ is unlikely to appear. The non-equilibrium wall function, on the other hand, is sensitized to pressure-gradient effects for mean velocity in log-law sublayer, which standard and proposed wall functions do not take into account. Therefore comparison and evaluation of the proposed three-zonal wall function only with standard wall function was carried out on the basis of backward-facing step experimental data from Driver's and Seegmiller's research [7].

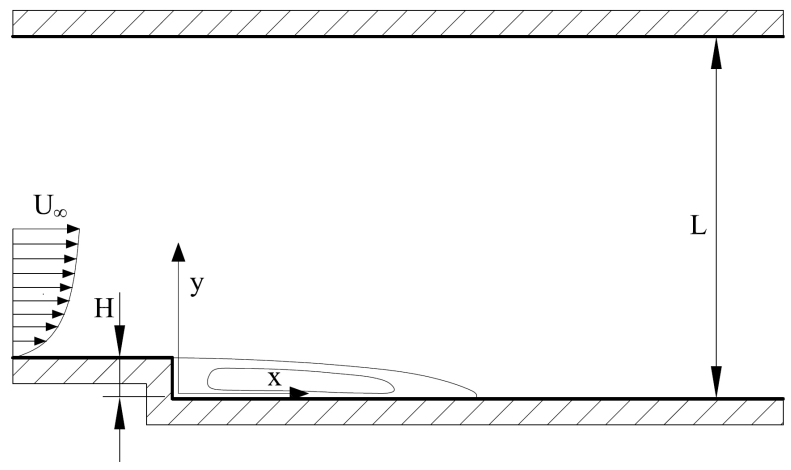

Fig. 3. Backward-facing step

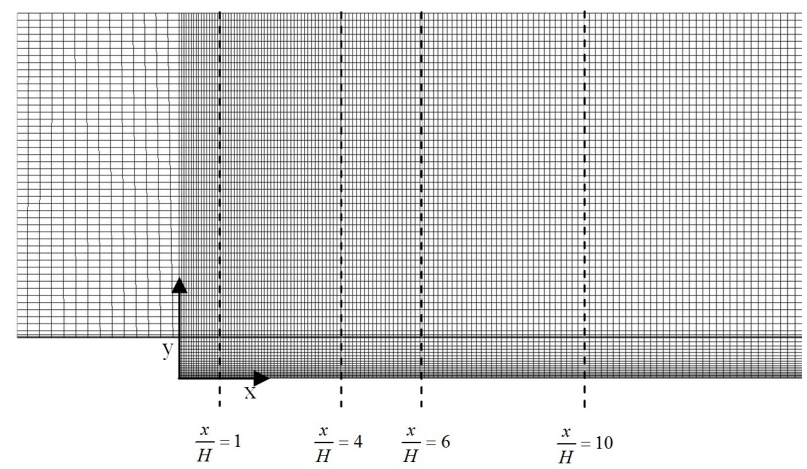

Fig. 4. Computational grid for CFD calculations

The boundary condition has been established to be the same as for experiment: $M_{r e f}=0.128, T_{r e f}=298$, at outlet $P / P_{\text {ref }}=1.011$. The computational grid shown in Fig. 4 was so adjusted that its first node in the grid was located in the buffer sublayer. The $y^{+}$achieved on this grid was in the range of $17.1-17.2$ (depending on different results from the use of individual wall functions).

\section{Skin friction coefficient}

The skin friction coefficient is defined as:

$$
C_{f}=\frac{\tau_{w, x}}{\frac{1}{2} \rho U_{\infty}^{2}},
$$

where:

$\tau_{w, x}$ is the local wall shear stress in $\mathrm{x}$ direction,

$U_{\infty} \quad$ - freestream reference velocity $(43.54 \mathrm{~m} / \mathrm{s})$.

In Fig. 5, a comparison between commercially available standard wall function, three-zonal wall function and experimental data is shown. Based on this comparison it can be observed that the standard wall function gives very similar results. In Fig. 6, the skin friction coefficient difference between numerical calculations and experiments is shown. Slightly better results are obtained using a new, three-zonal wall function in the area of highest computational differences $(x / H \in\langle 2 ; 8))$, where the solution obtained using the proposed wall function is up to $4.7 \%$ better in comparison with the standard wall function. Strong compliance with experimental results for both investigated wall functions can be observed for $(x / H \in\langle 8,12\rangle)$.

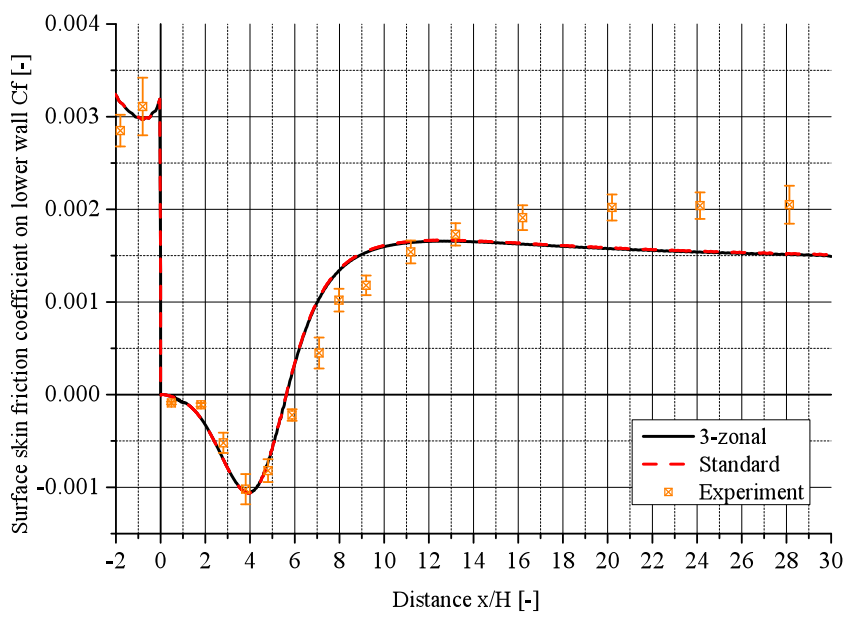

Fig. 5. Skin friction coefficient

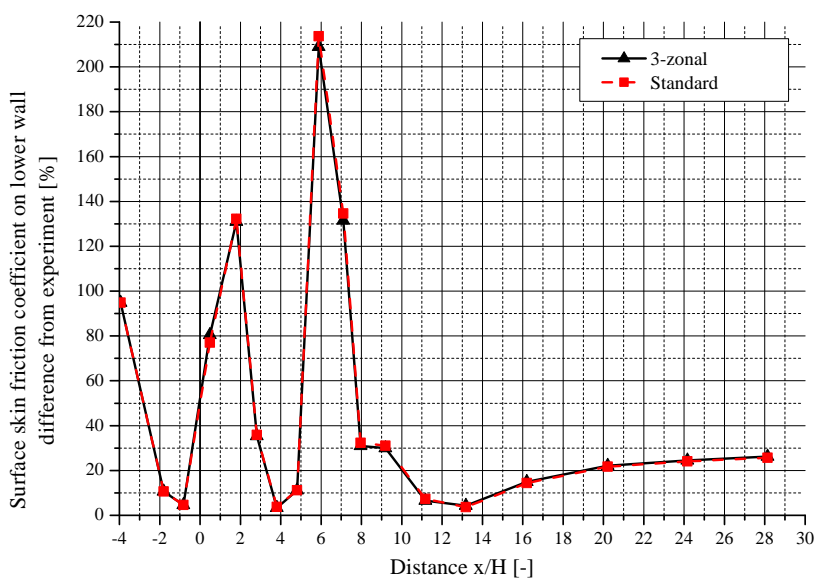

Fig. 6. Skin friction coefficient difference between numerical calculations and experiments 


\section{Surface pressure coefficient}

Fig. 7 shows a comparison of the surface pressure coefficient with experimental data and other near-wall approach models, which is defined as:

$$
C_{p}=\frac{p-p_{\infty}}{\frac{1}{2} \rho U_{\infty}^{2}}
$$



Fig. 7. Surface pressure coefficient

The nature of the surface pressure coefficient is consistent with experimental data; however, the quantitative results show a discrepancy for both functions. The maximum calculated relative error is up to $45 \%$. Better compliance of the model and experimental data is obtained for $x / H>9$, where differences are below $10 \%$. Calculation results between wall functions in this area vary by no more than $2 \%$.

\section{$\mathrm{U}$ velocities profiles}

In Fig. 8-15 $U$ velocity (velocity in $X$ direction) profiles on particular cross sections are shown. Sections are situated respectively in $x / H=1,4,6$ and 10 .

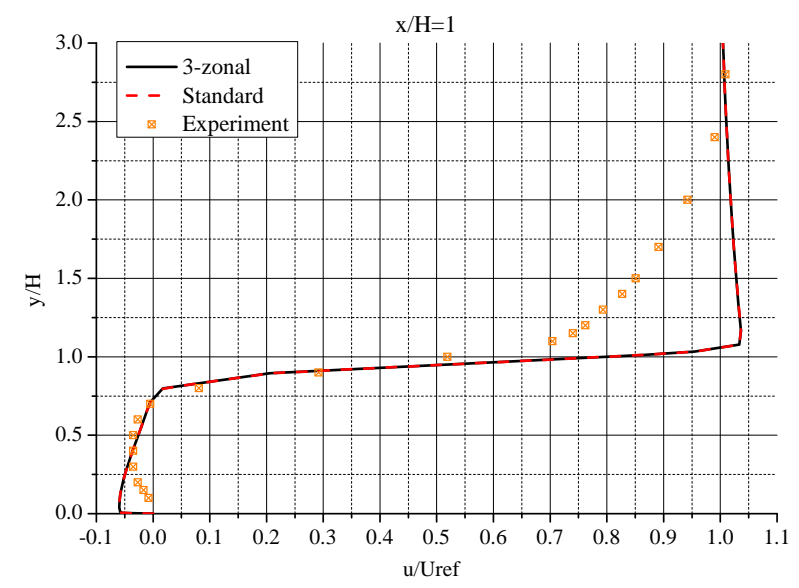

Fig. 8. U velocity profile for $x / H=1$

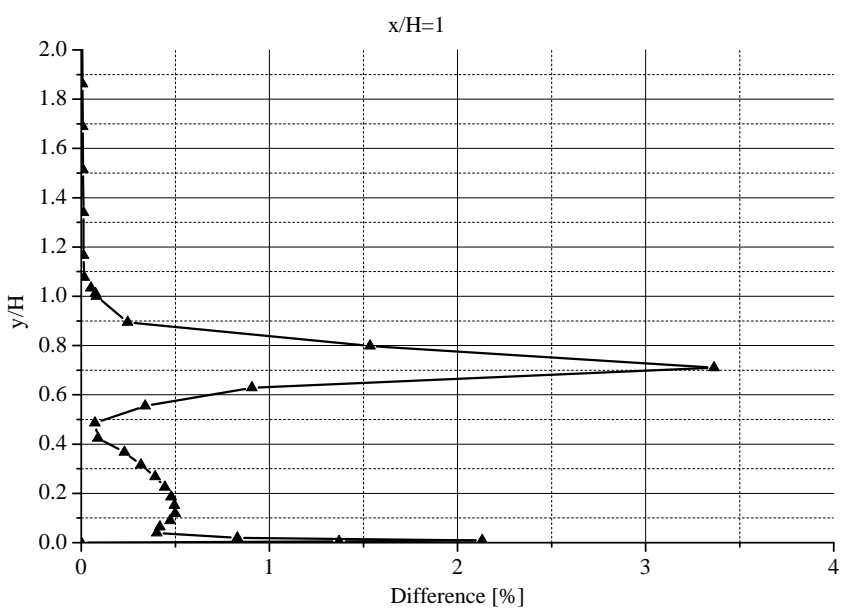

Fig. 9. Percentage difference of U velocity between 3-zonal and Standard wall function at $x / H=1$

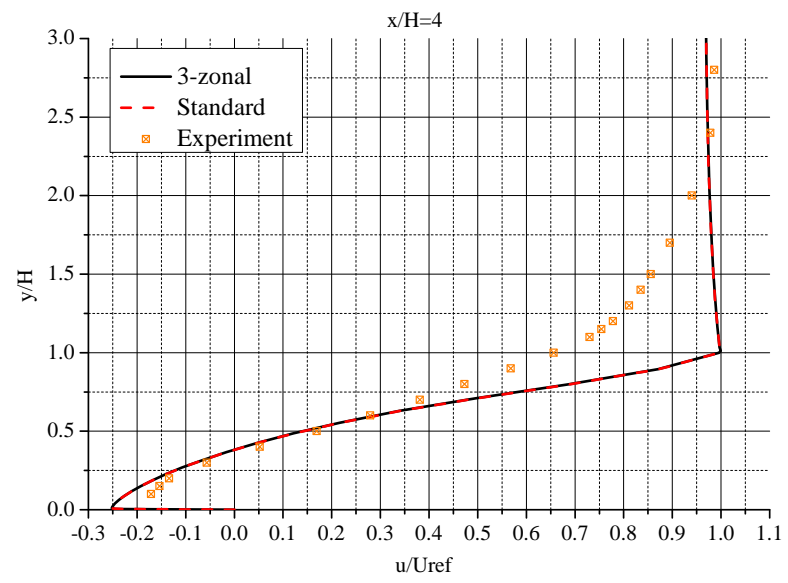

Fig. 10. U velocity profile for $x / H=4$

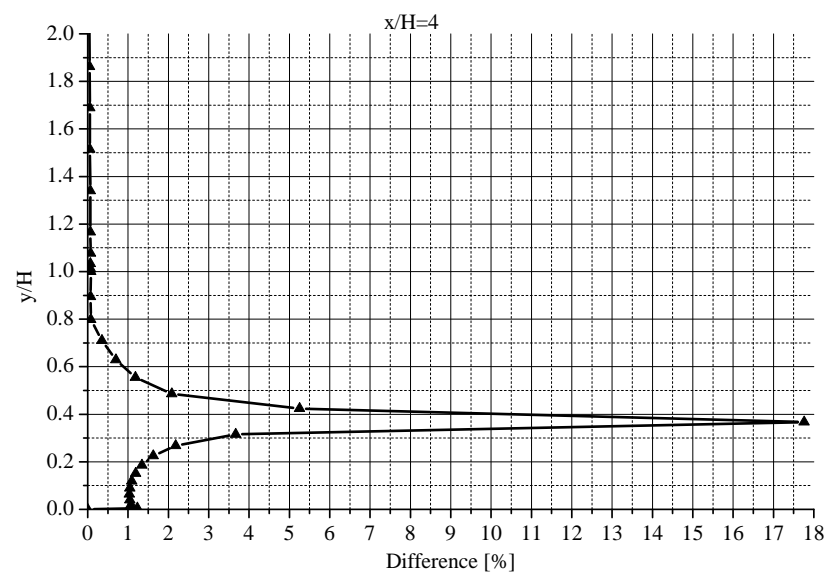

Fig. 11. Percentage difference of U velocity between 3-zonal and Standard wall function at $x / H=4$ 


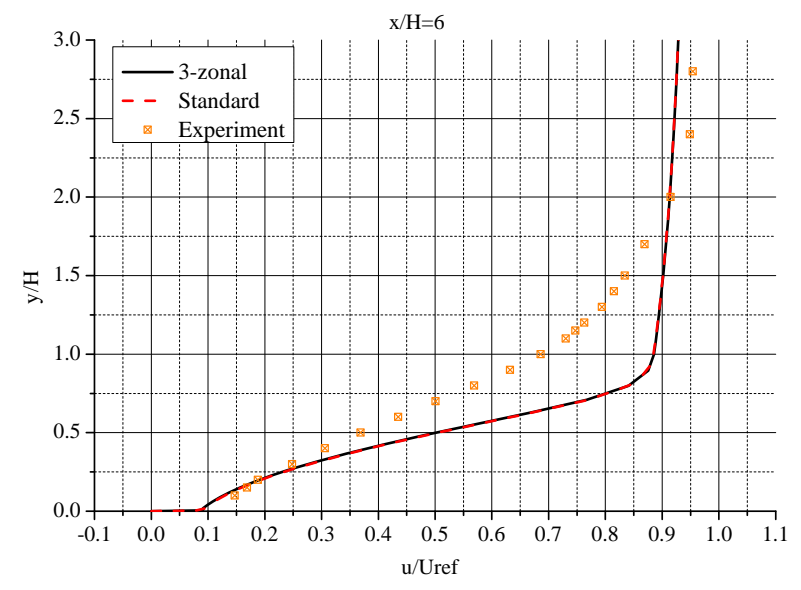

Fig. 12. U velocity profile for $x / H=6$

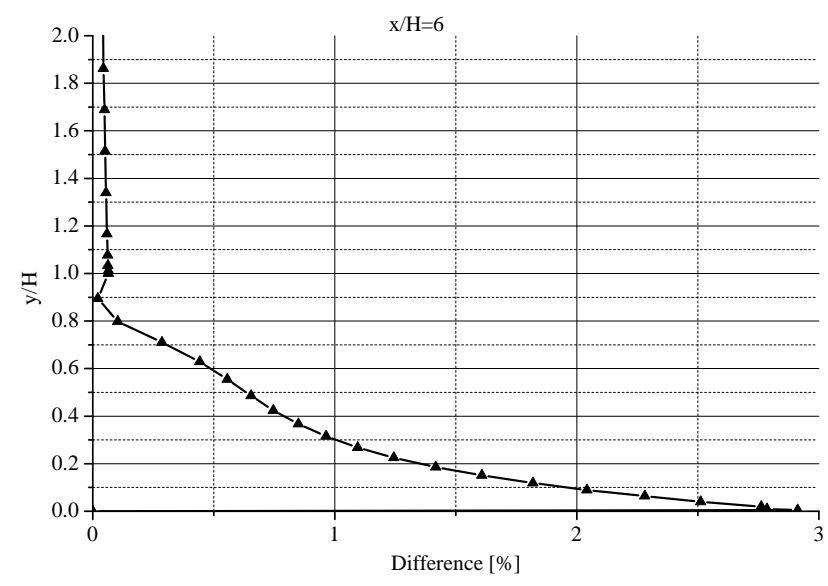

Fig. 13. Percentage difference of $U$ velocity between 3-zonal and Standard wall function at $x / H=6$

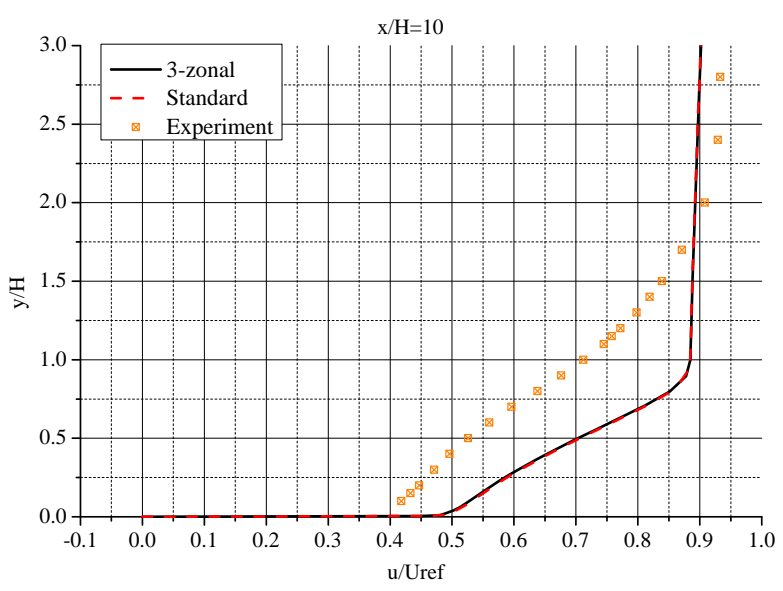

Fig. 14. $\mathrm{U}$ velocity profile for $\mathrm{x} / \mathrm{H}=10$
Calculated $U$ velocity profiles show good qualitative agreement with experimental data for both wall functions. Wall functions show small differences between each other. Greatest differences can be observed for $x / H=4$ (up to $17 \%$ ). For the rest of the cases differences are below $7 \%$. Above step height $y / H=1$ negligible differences between 3-zonal and standard wall function can be observed.

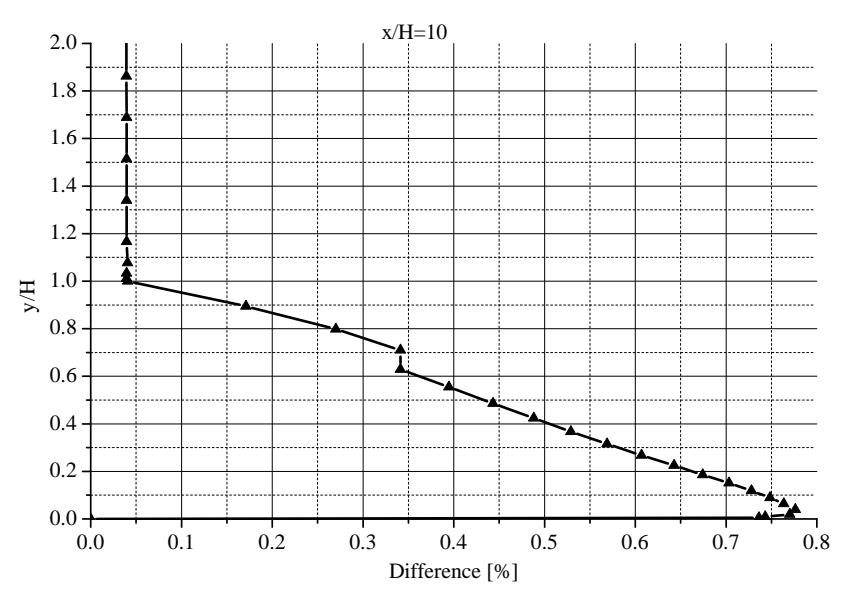

Fig. 15. Percentage difference of $U$ velocity between 3-zonal and Standard wall function at $x / H=10$

\section{CONCLUSION}

A new three-zonal wall function has been developed and implemented into the ANSYS FLUENT commercial CFD code. The wall function has been validated with the use of backward-facing step experimental data. The new wall function shows slightly better results (closer to experimental data) than the currently available standard wall functions for computational grids of $y^{+} \in\langle 5 ; 30)$, for the $\mathrm{k}-\varepsilon$ turbulence model. The wall function can be used in a wide range of numerical codes, adjusted to individual needs. The proposed approach can be useful in rapid calculations and provides a very good initial solution for more accurate numerical calculations of fluid flow parameters in devices with complex geometries, such as compressors, turbines and gas turbine combustors.

\section{References}

[1] J.O. Hinze, Turbulence, MacGraw-Hill 1975.

[2] K. Hanjalić , B.E. Lauder, Contribution towards a Reynoldsstress Closure for low-Reynolds number turbulence, J. Fluid Mech. 74, 593 (1976).

[3] H. Tennekes and J.L. Lumley, A first Course in Turbulence, MIT Press 1972.

[4] D.B. Spalding, A Single Formula for the "Law of the Wall", J. Appl. Mech. 28(3), 455 (1961). 
[5] T.H. Shih, L.A. Povinelli, N.S. Liu, M.G. Potapczuk and J.L. Lumley, A Generalized Wall Function, NASA Technical Memorandum - 1999-209398 (1999).

[6] D.M. Driver and H.L. Seegmiller, Backward-facing step with inclined opposite wall-experiments by Driver and Seegmiller, 1985.

[7] D.M. Driver and H.L. Seegmiller, Features of a reattachment turbulent shear layer in divergent channel flow. AIAA J. 23(2), 163-171 (1985).

[8] ANSYS, Inc., ANSYS FLUENT 14 Theory Guide, November 2011.

[9] ANSYS, Inc., ANSYS FLUENT 14 UDF Manual, November 2011.

[10] B.E. Launder and D.B. Spalding, The Numerical Computation of Turbulent Flows, Computer Methods in Applied Mechanics and Engineering 3, 269-289 (1974).
[11] D.C. Wilcox, Turbulence Modeling for CFD, DCW Industries Inc: La Canada, CA, 1998.

[12] V.C. Patel, W. Rodi and Scheuerer, Turbulence models for near-wall and low Reynolds numbers flows: A review, AIAA J. 23(9), 1308-1319 (1985).

[13] R. Absi, A simple eddy viscosity formulation for turbulent boundary layers near smooth walls, C. R. Mecanique 337, 158-165 (2009).

[14] 2D Backward Facing Step, NASA Langley Research Center, http://turbmodels.larc.nasa.gov/backstep_val.html, August 2012.
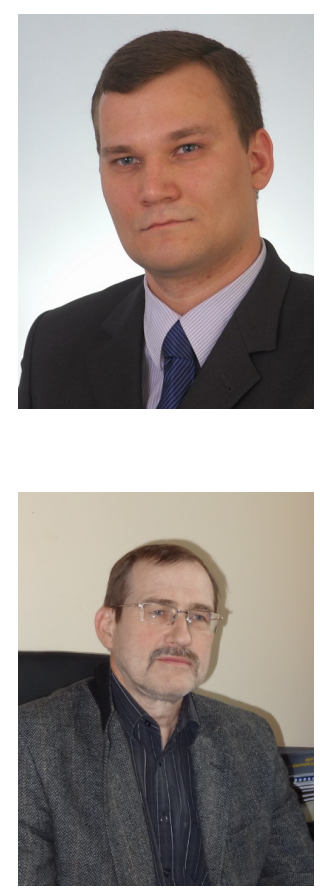

Maciej Chmielewski was born in 1988 in Łódź, Poland. He graduated from Warsaw University of Technology (WUT) in 2012 with an MSc degree in Aviation Engineering. He is currently a PhD student at the Institute of Heat Engineering, WUT. He works for Engineering Design Center (Institute of Aviation \& General Electric Company). His field of interests covers combustion chambers of turbine engines and numerical combustion modeling.

Marian Gieras professor at Warsaw University of Technology, Faculty of Power and Aeronautical Engineering. Degrees: PhD - 1987, DSc - 2006, Prof. - 2009, Warsaw University of Technology. His scientific specializations are Mechanics as well as Mechanical and Environmental Engineering. He has been member of the Polish Combustion Institute since 1994, and the Combustion Section of Thermodynamics and Combustion Committee of the Polish Academy of Sciences since 2008. Project manager and lead performer of several EU-granted projects. 